\title{
HALVES OF POINTS OF AN ODD DEGREE HYPERELLIPTIC CURVE IN ITS JACOBIAN
}

\author{
YURI G. ZARHIN
}

\begin{abstract}
Let $f(x)$ be a degree $(2 g+1)$ monic polynomial with coefficients in an algebraically closed field $K$ with $\operatorname{char}(K) \neq 2$ and without repeated roots. Let $\mathfrak{R} \subset K$ be the $(2 g+1)$-element set of roots of $f(x)$. Let $\mathcal{C}: y^{2}=f(x)$ be an odd degree genus $g$ hyperelliptic curve over $K$. Let $J$ be the jacobian of $\mathcal{C}$ and $J[2] \subset J(K)$ the (sub)group of points of order dividing 2. We identify $\mathcal{C}$ with the image of its canonical embedding into $J$ (the infinite point of $\mathcal{C}$ goes to the identity element of $J)$. Let $P=(a, b) \in \mathcal{C}(K) \subset J(K)$ and

$$
M_{1 / 2, P}=\{\mathfrak{a} \in J(K) \mid 2 \mathfrak{a}=P\} \subset J(K),
$$

which is $J[2]$-torsor. In a previous work we established an explicit bijection between the sets $M_{1 / 2, P}$ and$$
\mathfrak{R}_{1 / 2, P}:=\left\{\mathfrak{r}: \mathfrak{R} \rightarrow K \mid \mathfrak{r}(\alpha)^{2}=a-\alpha \forall \alpha \in \mathfrak{R} ; \prod_{\alpha \in \mathfrak{R}} \mathfrak{r}(\alpha)=-b\right\} .
$$

The aim of this paper is to describe the induced action of $J[2]$ on $\Re_{1 / 2, P}$ (i.e., how signs of square roots $r(\alpha)=\sqrt{a-\alpha}$ should change).
\end{abstract}

\section{INTRODUCTION}

Let $K$ be an algebraically closed field of characteristic different from $2, g$ a positive integer, $\mathfrak{R} \subset K$ a $(2 g+1)$-element set,

$$
f(x)=f_{\mathfrak{R}}(x):=\prod_{\alpha \in \mathfrak{R}}(x-\alpha)
$$

a degree $(2 g+1)$ polynomial with coefficients in $K$ and without repeated roots, $\mathcal{C}: y^{2}=f(x)$ the corresponding genus $g$ hyperelliptic curve over $K$, and $J$ the jacobian of $\mathcal{C}$. We identify $\mathcal{C}$ with the image of its canonical embedding

$$
\mathcal{C} \hookrightarrow J, P \mapsto \operatorname{cl}((P)-(\infty))
$$

into $J$ (the infinite point $\infty$ of $\mathcal{C}$ goes to the identity element of $J$ ). Let $J[2] \subset J(K)$ be the kernel of multiplication by 2 in $J(K)$, which is a $2 g$-dimensional $\mathbb{F}_{2}$-vector space. All the $(2 g+1)$ points

$$
\mathfrak{W}_{\alpha}:=(\alpha, 0) \in \mathcal{C}(K) \subset J(K)(\alpha \in \mathfrak{R})
$$

2010 Mathematics Subject Classification. 14H40, 14G27, 11G10.

Key words and phrases. Hyperelliptic curves, jacobians, Mumford representations.

Partially supported by Simons Foundation Collaboration grant \# 585711.

This paper was started during my stay in May-July 2018 at the Max-Planck-Institut für Mathematik (Bonn, Germany), whose hospitality and support are gratefully acknowledged. 
lie in $J[2]$ and generate it as the $2 g$-dimensional $\mathbb{F}_{2}$-vector space; they satisfy the only relation

$$
\sum_{\alpha \in \mathfrak{R}} \mathfrak{W}_{\alpha}=0 \in J[2] \subset J(K) .
$$

This leads to a well known canonical isomorphism [4] between $\mathbb{F}_{2}$-vector spaces $J[2]$ and

$$
\left(\mathbb{F}_{2}^{\mathfrak{R}}\right)^{0}=\left\{\phi: \mathfrak{R} \rightarrow \mathbb{F}_{2} \mid \sum_{\alpha \in \mathfrak{R}} \phi(\alpha)=0\right\} .
$$

Namely, each function $\phi \in\left(\mathbb{F}_{2}{ }^{\Re}\right)^{0}$ corresponds to

$$
\sum_{\alpha \in \mathfrak{R}} \phi(\alpha) \mathfrak{W}_{\alpha} \in J[2] .
$$

For example, for each $\beta \in \mathfrak{R}$ the point $\mathfrak{W}_{\beta}=\sum_{\alpha \neq \beta} \mathfrak{W}_{\alpha}$ corresponds to the function $\psi_{\beta}: \mathfrak{R} \rightarrow \mathbb{F}_{2}$ that sends $\beta$ to 0 and all other elements of $\mathfrak{R}$ to 1 .

If $\mathfrak{b} \in J(K)$ then the finite set

$$
M_{1 / 2, \mathfrak{b}}:=\{\mathfrak{a} \in J(K) \mid 2 \mathfrak{a}=\mathfrak{b}\} \subset J(K)
$$

consists of $2^{2 g}$ elements and carries the natural structure of a $J[2]$-torsor.

Let

$$
P=(a, b) \in \mathcal{C}(K) \subset J(K) .
$$

Let us consider, the set

$$
\mathfrak{R}_{1 / 2, P}:=\left\{\mathfrak{r}: \mathfrak{R} \rightarrow K \mid \mathfrak{r}(\alpha)^{2}=a-\alpha \forall \alpha \in \mathfrak{R} ; \prod_{\alpha \in \mathfrak{R}} \mathfrak{r}(\alpha)=-b\right\} .
$$

Changes of signs in the (even number of) square roots provide $\mathfrak{R}_{1 / 2, P}$ with the natural structure of a $\left(\mathbb{F}_{2}^{\Re}\right)^{0}$-torsor. Namely, let

$$
\chi: \mathbb{F}_{2} \rightarrow K^{*}
$$

be the additive character such that

$$
\chi(0)=1, \chi(1)=-1 \text {. }
$$

Then the result of the action of a function $\phi: \mathfrak{R} \rightarrow \mathbb{F}_{2}$ from $\left(\mathbb{F}_{2}{ }^{\mathfrak{R}}\right)^{0}$ on $\mathfrak{r}: \mathfrak{R} \rightarrow K$ from $\mathfrak{R}_{1 / 2, P}$ is just the product

$$
\chi(\phi) \mathfrak{r}: \mathfrak{R} \rightarrow K, \alpha \mapsto \chi(\phi(\alpha)) \mathfrak{r}(\alpha) .
$$

On the other hand, I constructed in [9] an explicit bijection of finite sets

$$
\mathfrak{R}_{1 / 2, P} \cong M_{1 / 2, P}, \mathfrak{r} \mapsto \mathfrak{a}_{\mathfrak{r}} \in M_{1 / 2, P} \subset J(K) .
$$

Identifying (as above) $J[2]$ and $\left(\mathbb{F}_{2}^{\Re}\right)^{0}$, we obtain a second structure of a $\left(\mathbb{F}_{2}^{\Re}\right)^{0}$ torsor on $\mathfrak{R}_{1 / 2, P}$. Our main result asserts that these two structures actually coincide. In down-to-earth terms this means the following.

Theorem 1.1. Let $\mathfrak{r} \in \mathfrak{R}_{1 / 2, P}$ and $\beta \in \mathfrak{R}$. Let us define $\mathfrak{r}^{\beta} \in \mathfrak{R}_{1 / 2, P}$ as follows.

$$
\mathfrak{r}^{\beta}(\beta)=\mathfrak{r}(\beta), \mathfrak{r}^{\beta}(\alpha)=-\mathfrak{r}(\alpha) \forall \alpha \in \mathfrak{R} \backslash\{\beta\} .
$$

Then

$$
\mathfrak{a}_{\mathfrak{r}^{\beta}}=\mathfrak{a}_{\mathfrak{r}}+\mathfrak{W}_{\beta}=\mathfrak{a}_{\mathfrak{r}}+\left(\sum_{\alpha \neq \beta} \mathfrak{W}_{\alpha}\right)
$$


Remark 1.2. In the case of elliptic curves (i.e., when $g=1$ ) the assertion of Theorem 1.1 was proven in [2, Th. 2.3(iv)].

Example 1.3. If $P=\mathfrak{W}_{\beta}=(\beta, 0)$ then

$$
\mathfrak{a}_{\mathfrak{r}}+\mathfrak{W}_{\beta}=\mathfrak{a}_{\mathfrak{r}}-\mathfrak{W}_{\beta}=\mathfrak{a}_{\mathfrak{r}}-2 \mathfrak{a}_{\mathfrak{r}}=-\mathfrak{a}_{\mathfrak{r}}
$$

while

$$
-\mathfrak{a}_{\mathfrak{r}}=\mathfrak{a}_{-\mathfrak{r}}
$$

(see $[9$, Remark 3.5]). On the other hand, $\mathfrak{r}(\beta)=\sqrt{\beta-\beta}=0$ for all $\mathfrak{r}$ and

$$
\mathfrak{r}^{\beta}=-\mathfrak{r}: \alpha \mapsto-\mathfrak{r}(\alpha) \forall \alpha \in \mathfrak{R} .
$$

This implies that

$$
\mathfrak{a}_{\mathfrak{r}^{\beta}}=\mathfrak{a}_{-\mathfrak{r}}=\mathfrak{a}_{\mathfrak{r}}+\mathfrak{W}_{\beta} .
$$

This proves Theorem 1.1 in the special case $P=\mathfrak{W}_{\beta}$.

The paper is organized as follows. In Section 2 we recall basic facts about Mumford representations of points of $J(K)$ and review results of [9], including an explicit description of the bijection between $\Re_{1 / 2, P}$ and $M_{1 / 2, P}$. In Section 3 we give explicit formulas for the Mumford representation of $\mathfrak{a}+\mathfrak{W}_{\beta}$ when $\mathfrak{a}$ lies neither on the theta divisor of $J$ nor on its translation by $\mathfrak{W}_{\beta}$, assuming that we know the Mumford representation of $\mathfrak{a}$. In Section 4 we prove Theorem 1.1, using auxiliary results from commutative algebra that are proven in Section 5 .

\section{HALVES AND SQUARE ROOTS}

Let $\mathcal{C}$ be the smooth projective model of the smooth affine plane $K$-curve

$$
y^{2}=f(x)=\prod_{\alpha \in \mathfrak{R}}(x-\alpha)
$$

where $\mathfrak{R}$ is a $(2 g+1)$-element subset of $K$. In particular, $f(x)$ is a monic degree $(2 g+1)$ polynomial without repeated roots. It is well known that $\mathcal{C}$ is a genus $g$ hyperelliptic curve over $K$ with precisely one infinite point, which we denote by $\infty$. In other words,

$$
\mathcal{C}(K)=\left\{(a, b) \in K^{2} \mid b^{2}=\prod_{\alpha \in \Re}\left(a-\alpha_{i}\right)\right\} \bigsqcup\{\infty\} .
$$

Clearly, $x$ and $y$ are nonconstant rational functions on $\mathcal{C}$, whose only pole is $\infty$. More precisely, the polar divisor of $x$ is $2(\infty)$ and the polar divisor of $y$ is $(2 g+1)(\infty)$. The zero divisor of $y$ is $\sum_{\alpha \in \mathfrak{R}}\left(\mathfrak{W}_{\alpha}\right)$. In particular, $y$ is a local parameter at (every) $\mathfrak{W}_{\alpha}$.

We write $\iota$ for the hyperelliptic involution

$$
\iota: \mathcal{C} \rightarrow \mathcal{C},(x, y) \mapsto(x,-y), \infty \mapsto \infty .
$$

The set of fixed points of $\iota$ consists of $\infty$ and all $\mathfrak{W}_{\alpha}(\alpha \in \mathfrak{R})$. It is well known that for each $P \in \mathcal{C}(K)$ the divisor $(P)+\iota(P)-2(\infty)$ is principal. More precisely, if $P=(a, b) \in \mathcal{C}(K)$ then $(P)+\iota(P)-2(\infty)$ is the divisor of the rational function $x-a$ on $C$. In particular, if $P=\mathfrak{W}_{\alpha}=(\alpha, 0)$ then

$$
2\left(\mathfrak{W}_{\alpha}\right)-2(\infty)=\operatorname{div}(x-\alpha) .
$$

In particular, $x-\alpha$ has a double zero at $\mathfrak{W}_{\alpha}$ (and no other zeros). If $D$ is a divisor on $\mathcal{C}$ then we write $\operatorname{supp}(D)$ for its support, which is a finite subset of $\mathcal{C}(K)$. 
Recall that the jacobian $J$ of $\mathcal{C}$ is a $g$-dimensional abelian variety over $K$. If $D$ is a degree zero divisor on $\mathcal{C}$ then we write $\operatorname{cl}(D)$ for its linear equivalence class, which is viewed as an element of $J(K)$. Elements of $J(K)$ may be described in terms of so called Mumford representations (see [4, Sect. 3.12], [8, Sect. 13.2] and Subsection 2.3 below).

We will identify $\mathcal{C}$ with its image in $J$ with respect to the canonical regular map $\mathcal{C} \hookrightarrow J$ under which $\infty$ goes to the identity element of $J$. In other words, a point $P \in \mathcal{C}(K)$ is identified with $\operatorname{cl}((P)-(\infty)) \in J(K)$. Then the action of the hyperelliptic involution $\iota$ on $\mathcal{C}(K) \subset J(K)$ coincides with multiplication by -1 on $J(K)$. In particular, the list of points of order 2 on $\mathcal{C}$ consists of all $\mathfrak{W}_{\alpha}(\alpha \in \mathfrak{R})$.

2.1. Since $K$ is algebraically closed, the commutative group $J(K)$ is divisible. It is well known that for each $\mathfrak{b} \in J(K)$ there are exactly $2^{2 g}$ elements $\mathfrak{a} \in J(K)$ such that $2 \mathfrak{a}=\mathfrak{b}$. In [9] we established explicitly the following bijection $\mathfrak{r} \mapsto \mathfrak{a}_{\mathfrak{r}}$ between the $2^{2 g}$-element sets $\Re_{1 / 2, P}$ and $M_{1 / 2, P}$.

If $\mathfrak{r} \in \mathfrak{R}_{1 / 2, P}$ then for each positive integer $i \leq 2 g+1$ let us consider $\mathbf{s}_{i}(\mathfrak{r}) \in K$ defined as the value of $i$ th basic symmetric function at $(2 g+1)$ elements $\{\mathfrak{r}(\alpha) \mid \alpha \in$ $\mathfrak{R}\}$ (notice that all $\mathfrak{r}(\alpha)$ are distinct, since their squares $\mathfrak{r}(\alpha)^{2}=a-\alpha$ are distinct). Let us consider the degree $g$ monic polynomial

$$
U_{\mathfrak{r}}(x)=(-1)^{g}\left[(a-x)^{g}+\sum_{j=1}^{g} \mathbf{s}_{2 j}(\mathfrak{r})(a-x)^{g-j}\right],
$$

and the polynomial

$$
V_{\mathfrak{r}}(x)=\sum_{j=1}^{g}\left(\mathbf{s}_{2 j+1}(\mathfrak{r})-\mathbf{s}_{1}(\mathfrak{r}) \mathbf{s}_{2 j}(\mathfrak{r})\right)(a-x)^{g-j}
$$

whose degree is strictly less than $g$. Let $\left\{c_{1}, \ldots, c_{g}\right\} \subset K$ be the collection of all $g$ roots of $U_{\mathfrak{r}}(x)$, i.e.,

$$
U_{\mathfrak{r}}(x)=\prod_{j=1}^{g}\left(x-c_{j}\right) \in K[x] .
$$

Let us put

$$
d_{j}=V_{\mathfrak{r}}\left(c_{j}\right) \forall j=1, \ldots, g .
$$

It is proven in $[9$, Th. 3.2$]$ that $Q_{j}=\left(c_{j}, d_{j}\right)$ lies in $\mathcal{C}(K)$ for all $j$ and

$$
\mathfrak{a}_{\mathfrak{r}}:=\mathrm{cl}\left(\left(\sum_{j=1}^{g}\left(Q_{j}\right)\right)-g(\infty)\right) \in J(K)
$$

satisfies $2 \mathfrak{a}_{\mathfrak{r}}=P$, i.e., $\mathfrak{a}_{\mathfrak{r}} \in M_{1 / 2, P}$. In addition, none of $Q_{j}$ coincides with any $\mathfrak{W}_{\alpha}$, i.e.,

$$
U_{\mathfrak{r}}(\alpha) \neq 0, c_{j} \neq \alpha, d_{j} \neq 0 .
$$

The main result of [9] asserts that the map

$$
\mathfrak{R}_{1 / 2, P} \rightarrow M_{1 / 2, P}, \mathfrak{r} \mapsto \mathfrak{a}_{\mathfrak{r}}
$$

is a bijection. 
Remark 2.2. Notice that one may express explicitly $\mathfrak{r}$ in terms of $U_{\mathfrak{r}}(x)$ and $V_{\mathfrak{r}}(x)$. Namely [9, Th. 3.2], none of $\alpha \in \mathfrak{R}$ is a root of $U_{\mathfrak{r}}(x)$ and

$$
\mathfrak{r}(\alpha)=\mathbf{s}_{1}(\mathfrak{r})+(-1)^{g} \frac{V_{\mathfrak{r}}(\alpha)}{U_{\mathfrak{r}}(\alpha)} \text { for all } \alpha \in \mathfrak{R}
$$

In order to determine $\mathbf{s}_{1}(\mathfrak{r})$, let us fix two distinct roots $\beta, \gamma \in \mathfrak{R}$. Then [9, Cor. $3.4]$

and

$$
\frac{V_{\mathfrak{r}}(\gamma)}{U_{\mathfrak{r}}(\gamma)} \neq \frac{V_{\mathfrak{r}}(\beta)}{U_{\mathfrak{r}}(\beta)}
$$

$$
\mathbf{s}_{1}(\mathfrak{r})=\frac{(-1)^{g}}{2} \times \frac{\left(\beta+\left(\frac{V_{\mathfrak{r}}(\beta)}{U_{\mathfrak{r}}(\beta)}\right)^{2}\right)-\left(\gamma+\left(\frac{V_{\mathbf{r}}(\gamma)}{U_{\mathbf{r}}(\gamma)}\right)^{2}\right)}{\frac{V_{\mathfrak{r}}(\gamma)}{U_{\mathfrak{r}}(\gamma)}-\frac{V_{\mathbf{r}}(\beta)}{U_{\mathfrak{r}}(\beta)}} .
$$

2.3. Mumford representations (see [4, Sect. 3.12], [8, Sect. 13.2, pp. 411-415, especially, Prop. 13.4, Th. 13.5 and Th. 13.7]). Recall [8, Sect. 13.2, p. 411] that if $D$ is an effective divisor on $\mathcal{C}$ of (nonnegative) degree $m$, whose support does not contain $\infty$, then the degree zero divisor $D-m(\infty)$ is called semi-reduced if it enjoys the following properties.

- If $\mathfrak{W}_{\alpha}$ lies in $\operatorname{supp}(D)$ then it appears in $D$ with multiplicity 1 .

- If a point $Q$ of $\mathcal{C}(K)$ lies in $\operatorname{supp}(D)$ and does not coincide with any of $\mathfrak{W}_{\alpha}$ then $\iota(Q)$ does not lie in $\operatorname{supp}(D)$.

If, in addition, $m \leq g$ then $D-m(\infty)$ is called reduced.

It is known ([4, Ch. 3a], [8, Sect. 13.2, Prop. 3.6 on p. 413]) that for each $\mathfrak{a} \in J(K)$ there exist exactly one nonnegative $m$ and (effective) degree $m$ divisor $D$ such that the degree zero divisor $D-m(\infty)$ is reduced and $\operatorname{cl}(D-m(\infty))=\mathfrak{a}$. If

$$
m \geq 1, D=\sum_{j=1}^{m}\left(Q_{j}\right) \text { where } Q_{j}=\left(a_{j}, b_{j}\right) \in \mathcal{C}(K) \text { for all } j=1, \ldots, m
$$

(here $Q_{j}$ do not have to be distinct) then the corresponding

$$
\mathfrak{a}=\operatorname{cl}(D-m(\infty))=\sum_{j=1}^{m} Q_{j} \in J(K) .
$$

The Mumford representation of $\mathfrak{a} \in J(K)$ is the pair $(U(x), V(x))$ of polynomials $U(x), V(x) \in K[x]$ such that

$$
U(x)=\prod_{j=1}^{m}\left(x-a_{j}\right)
$$

is a degree $m$ monic polynomial while $V(x)$ has degree $<m=\operatorname{deg}(U)$, the polynomial $V(x)^{2}-f(x)$ is divisible by $U(x)$, and

$$
b_{j}=V\left(a_{j}\right), Q_{j}=\left(a_{j}, V\left(a_{j}\right)\right) \in \mathcal{C}(K) \text { for all } j=1, \ldots m .
$$

(Here $\left(a_{j}, b_{j}\right)$ are as above.) Such a pair always exists, is unique, and (as we have just seen) uniquely determines not only $\mathfrak{a}$ but also divisors $D$ and $D-m(\infty)$.

Conversely, if $U(x)$ is a monic polynomial of degree $m \leq g$ and $V(x)$ a polynomial such that $\operatorname{deg}(V)<\operatorname{deg}(U)$ and $V(x)^{2}-f(x)$ is divisible by $U(x)$ then there exists exactly one $\mathfrak{a}=\operatorname{cl}(D-m(\infty))$ where $D-m(\infty)$ is a reduced divisor and $(U(x), V(x))$ is the Mumford representation of $\mathfrak{a}=\operatorname{cl}(D-m(\infty))$. 
2.4. In the notation of Subsect. 2.1, let us consider the effective degree $g$ divisor

$$
D_{\mathfrak{r}}:=\sum_{j=1}\left(Q_{j}\right)
$$

on $\mathcal{C}$. Then $\operatorname{supp}\left(D_{\mathfrak{r}}\right)$ (obviously) does contain neither $\infty$ nor any of $\mathfrak{W}_{\alpha}$ 's. It is proven in $\left[9\right.$, Th. 3.2] that the divisor $D_{\mathfrak{r}}-g(\infty)$ is reduced and the pair $\left(U_{\mathfrak{r}}(x), V_{\mathfrak{r}}(x)\right)$ is the Mumford representation of

$$
\mathfrak{a}_{\mathfrak{r}}:=\operatorname{cl}\left(D_{\mathfrak{r}}-g(\infty)\right) .
$$

In particular, if $Q \in C(K)$ lies in $\operatorname{supp}(D)$ (i.e., is one of $Q_{j}$ 's) then $\iota(Q)$ does not.

Lemma 2.5. Let $D$ be an effective divisor on $\mathcal{C}$ of degree $m>0$ such that $m \leq 2 g+1$ and $\operatorname{supp}(D)$ does not contain $\infty$. Assume that the divisor $D-m(\infty)$ is principal.

(1) Suppose that $m$ is odd. Then:

(i) $m=2 g+1$ and there exists exactly one polynomial $v(x) \in K[x]$ such that the divisor of $y-v(x)$ coincides with $D-(2 g+1)(\infty)$. In addition, $\operatorname{deg}(v) \leq g$.

(ii) If $\mathfrak{W}_{\alpha}$ lies in $\operatorname{supp}(D)$ then it appears in $D$ with multiplicity 1.

(iii) If $b$ is a nonzero element of $K$ and $P=(a, b) \in \mathcal{C}(K)$ lies in $\operatorname{supp}(D)$ then $\iota(P)=(a,-b)$ does not lie in $\operatorname{supp}(D)$.

(iv) $D-(2 g+1)(\infty)$ is semi-reduced (but not reduced).

(2) Suppose that $m=2 d$ is even. Then:

(i) there exists exactly one monic degree d polynomial $u(x) \in K[x]$ such that the divisor of $u(x)$ coincides with $D-m(\infty)$;

(ii) every point $Q \in \mathcal{C}(K)$ appears in $D-m(\infty)$ with the same multiplicity as $\iota(Q)$;

(iii) every $\mathfrak{W}_{\alpha}$ appears in $D-m(\infty)$ with even multiplicity.

Proof. All the assertions except (2)(iii) are already proven in [9, Lemma 2.2]. In order to prove the remaining one, let us split the polynomial $v(x)$ into a product $v(x)=(x-\alpha)^{d} v_{1}(x)$ where $d$ is a nonnegative integer and $v_{1}(x) \in K[x]$ satisfies $v_{1}(\alpha) \neq 0$. Then $\mathfrak{W}_{\alpha}$ appears in $D-m(\infty)$ with multiplicy $2 d$, because $(x-\alpha)$ has a double zero at $\mathfrak{W}_{\alpha}$. (See also [5].)

Let $d \leq g$ be a positive integer and $\Theta_{d} \subset J$ be the image of the regular map

$$
\mathcal{C}^{d} \rightarrow J,\left(Q_{1}, \ldots, Q_{d}\right) \mapsto \sum_{i=1}^{d} Q_{i} \subset J
$$

It is well known that $\Theta_{d}$ is an irreducible closed $d$-dimensional subvariety of $J$ that coincides with $\mathcal{C}$ for $d=1$ and with $J$ if $d=g$; in addition, $\Theta_{d} \subset \Theta_{d+1}$ for all $d<g$. Clearly, each $\Theta_{d}$ is stable under multiplication by -1 in $J$. We write $\Theta$ for the $(g-1)$-dimensional theta divisor $\Theta_{g-1}$.

Theorem 2.6 (See Th. 2.5 of [9]). Suppose that $g>1$ and let

$$
\mathcal{C}_{1 / 2}:=2^{-1} \mathcal{C} \subset J
$$

be the preimage of $\mathcal{C}$ with respect to multiplication by 2 in $J$. Then the intersection of $\mathcal{C}_{1 / 2}(K)$ and $\Theta$ consists of points of order dividing 2 on $J$. In particular, the intersection of $\mathcal{C}$ and $\mathcal{C}_{1 / 2}$ consists of $\infty$ and all $\mathfrak{W}_{\alpha}$ 's. 


\section{Adding Weierstrass points}

In this section we discuss how to compute a sum $\mathfrak{a}+\mathfrak{W}_{\beta}$ in $J(K)$ when $\mathfrak{a} \in J(K)$ lies neither on $\Theta$ nor on its translation $\Theta+\mathfrak{W}_{\beta}$. Let $D-g(\infty)$ be the reduced divisor on $\mathcal{C}$, whose class represents $\mathfrak{a}$. Here

$$
D=\sum_{j=1}^{g}\left(Q_{j}\right) \text { where } Q_{j}=\left(a_{j}, b_{j}\right) \in \mathcal{C}(K) \backslash\{\infty\}
$$

is a degree $g$ effective divisor. Let $(U(x), V(x))$ be the Mumford representation of $\operatorname{cl}(D-g(\infty))$. We have

$$
\begin{gathered}
\operatorname{deg}(U)=g>\operatorname{deg}(V) \\
U(x)=\prod_{j=1}^{g}\left(x-a_{j}\right), b_{j}=V\left(a_{j}\right) \forall j
\end{gathered}
$$

and $f(x)-V(x)^{2}$ is divisible by $U(x)$.

Example 3.1. Assume additionally that none of $Q_{j}$ coincides with $\mathfrak{W}_{\beta}=(\beta, 0)$, i.e.,

$$
U(\beta) \neq 0
$$

Let us find explicitly the Mumford representation $\left(U^{[\beta]}(x), V^{[\beta]}(x)\right)$ of the sum $\mathfrak{a}+\mathfrak{W}_{\beta}=\operatorname{cl}(D-m(\infty))+\operatorname{cl}\left(\left(\mathfrak{W}_{\beta}\right)-(\infty)\right)=\operatorname{cl}\left(\left(D+\left(\mathfrak{W}_{\beta}\right)\right)-(g+1)(\infty)\right)=\operatorname{cl}\left(D_{1}-(g+1)(\infty)\right)$. where

$$
D_{1}:=D+\left(\mathfrak{W}_{\beta}\right)=\left(\sum_{j=1}^{g}\left(Q_{j}\right)\right)+\left(\mathfrak{W}_{\beta}\right)
$$

is a degree $(g+1)$ effective divisor on $\mathcal{C}$. (We will see that $\operatorname{deg}\left(\tilde{U}^{[\beta]}\right)=g$.) Clearly, $D_{1}-(g+1)(\infty)$ is semi-reduced but not reduced.

Let us consider the polynomials

$$
U_{1}(x)=(x-\beta) U(x), V_{1}(x)=V(x)-\frac{V(\beta)}{U(\beta)} U(x) \in K[x] .
$$

Then $U_{1}$ is a degree $(g+1)$ monic polynomial, $\operatorname{deg}\left(V_{1}\right) \leq g$,

$$
V_{1}(\beta)=0, V_{1}\left(a_{j}\right)=V\left(a_{j}\right)=b_{j} \forall j
$$

and $f(x)-V_{1}(x)^{2}$ is divisible by $U_{1}(x)$. (The last assertion follows from the divisibility of both $f(x)$ and $V_{1}(x)$ by $x-\beta$ combined with the divisibility of $f(x)-V(x)^{2}$ by $U(x)$.) If we put

$$
a_{g+1}=\beta, b_{g+1}=0, Q_{g+1}=\mathfrak{W}_{\beta}=(\beta, 0)
$$

then

$$
U_{1}(x)=\prod_{j=1}^{g+1}\left(x-a_{j}\right), D_{1}=\sum_{j=1}^{g+1}\left(Q_{j}\right) \text { where } Q_{j}=\left(a_{j}, b_{j}\right) \in \mathcal{C}(K), b_{j}=V_{1}\left(a_{j}\right) \forall j
$$

and $f(x)-V_{1}(x)^{2}$ is divisible by $U_{1}(x)$. In particular, $\left(U_{1}(x), V_{1}(x)\right)$ is the pair of polynomials that corresponds to semi-reduced $D_{1}-(g+1)(\infty)$ as described in [8, Prop. 13.4 and Th. 3.5]. In order to find the Mumford representation of 
$\operatorname{cl}\left(D_{1}-(g+1)(\infty)\right)$, we use an algorithm described in [8, Th. 13.9]. Namely, let us put

$$
\tilde{U}(x)=\frac{f(x)-V_{1}(x)^{2}}{U_{1}(x)} \in K[x] .
$$

Since $\operatorname{deg}\left(V_{1}(x)\right) \leq g$ and $\operatorname{deg}(f)=2 g+1$, we have

$$
\operatorname{deg}\left(V_{1}(x)^{2}\right) \leq 2 g, \operatorname{deg}\left(f(x)-V_{1}(x)^{2}\right)=2 g+1, \operatorname{deg}(\tilde{U}(x))=g .
$$

Since $f(x)$ is monic, $f(x)-V_{1}(x)^{2}$ is also monic and therefore $\tilde{U}(x)$ is also monic, because $U_{1}(x)$ is monic. By [8, Th. 13.9], $U^{[\beta]}(x)=\tilde{U}(x)$ (since the latter is monic and has degree $g \leq g)$ and $V^{[\beta]}(x)$ is the remainder of $-V_{1}(x)$ with respect to division by $\tilde{U}(x)$. Let us find this remainder. We have

$$
-V_{1}(x)=-\left(V(x)-\frac{V(\beta)}{U(\beta)} U(x)\right)=-V(x)+\frac{V(\beta)}{U(\beta)} U(x) .
$$

Recall that

$$
\operatorname{deg}(V)<g=\operatorname{deg}(U)=\operatorname{deg}(\tilde{U}) .
$$

This implies that the coefficient of $-V_{1}$ at $x^{g}$ equals $V(\beta) / U(\beta)$ and therefore

$$
V^{[\beta]}(x)=\left(-V(x)+\frac{V(\beta)}{U(\beta)} U(x)\right)-\frac{V(\beta)}{U(\beta)} \tilde{U}(x)=-V(x)+\frac{V(\beta)}{U(\beta)}(U(x)-\tilde{U}(x)) .
$$

Using formulas above for $U_{1}, V_{1}, \tilde{U}$, we obtain that

$$
\begin{gathered}
U^{[\beta]}(x)=\frac{f(x)-\left(V(x)-\frac{V(\beta)}{U(\beta)} U(x)\right)^{2}}{(x-\beta) U(x)}, \\
V^{[\beta]}(x)=-V(x)+\frac{V(\beta)}{U(\beta)}\left(U(x)-\frac{f(x)-\left(V(x)-\frac{V(\beta)}{U(\beta)} U(x)\right)^{2}}{(x-\beta) U(x)}\right) .
\end{gathered}
$$

Remark 3.2. There is an algorithm of David Cantor [8, Sect. 13.3] that explains how to compute the Mumford representation of a sum of arbitrary divisor classes (elements of $J(K))$ when their Mumford representations are given.

Remark 3.3. Suppose that $\mathfrak{a} \in J(K)$ and $P=2 \mathfrak{a}$ lies in $\mathcal{C}(K)$ but is not the zero of the group law. Then $\mathfrak{a}$ does not lie on the theta divisor (Theorem 2.6) and satisfies the conditions of Example 3.1 for all $\beta \in \mathfrak{R}$ (see Subsect. 2.1).

\section{Proof of Main Theorem}

Let us choose an order on $\mathfrak{R}$. This allows us to identify $\mathfrak{R}$ with $\{1, \ldots, 2 g, 2 g+1\}$ and list elements of $\mathfrak{R}$ as $\left\{\alpha_{1}, \ldots, \alpha_{2 g}, \alpha_{2 g+1}\right\}$. Then

$$
f(x)=\prod_{i=1}^{2 g+1}\left(x-\alpha_{i}\right)
$$

and the affine equation for $\mathcal{C} \backslash\{\infty\}$ is

$$
y^{2}=\prod_{i=1}^{2 g+1}\left(x-\alpha_{i}\right) .
$$

Slightly abusing notation, we denote $\mathfrak{W}_{\alpha_{i}}$ by $\mathfrak{W}_{i}$. 
Let us consider the closed affine $K$-subset $\tilde{\mathcal{C}}$ in the affine $K$-space $\mathbb{A}^{2 g+1}$ with coordinate functions $z_{1}, \ldots, z_{2 g}, z_{2 g+1}$ that is cut out by the system of quadratic equations

$$
z_{1}^{2}+\alpha_{1}=z_{2}^{2}+\alpha_{2}=\cdots=z_{2 g+1}^{2}+\alpha_{2 g+1}
$$

We write $x$ for the regular function $z_{i}^{2}+\alpha_{i}$ on $\tilde{\mathcal{C}}$, which does not depend on a choice of $i$. By Hilbert's Nullstellensatz, the $K$-algebra $K[\tilde{\mathcal{C}}]$ of regular functions on $\tilde{\mathcal{C}}$ is canonically isomorphic to the following $K$-algebra. First, we need to consider the quotient $A$ of the polynomial $K[x]$-algebra $K[x]\left[T_{1}, \ldots, T_{2 g+1}\right]$ by the ideal generated by all quadratic polynomials $T_{i}^{2}-\left(x-\alpha_{i}\right)$. Next, $K[\tilde{\mathcal{C}}]$ is canonically isomorphic to the quotient $A / \mathcal{N}(A)$ where $\mathcal{N}(A)$ is the nilradical of $A$. In the next section (Example 5.4) we will prove that $A$ has no zero divisors (in particular, $\mathcal{N}(A)=\{0\}$ ) and therefore $\tilde{\mathcal{C}}$ is irreducible. (See also [3].) We write $y$ for the regular function

$$
y=-\prod_{i=1}^{2 g} z_{i} \in K[\tilde{\mathcal{C}}]
$$

Clearly, $y^{2}=\prod_{i=1}^{2 g}\left(x-\alpha_{i}\right)$ in $K[\tilde{\mathcal{C}}]$. The pair $(x, y)$ gives rise to the finite regular map of affine $K$-varieties (actually, curves)

$$
\mathfrak{h}: \tilde{\mathcal{C}} \rightarrow \mathcal{C} \backslash\{\infty\},\left(r_{1}, \ldots, r_{2 g}, r_{2 g+1}\right) \mapsto(a, b)=\left(r_{1}^{2}+\alpha_{1},-\prod_{i=1}^{2 g+1} r_{i}\right)
$$

of degree $2^{2 g}$. For each

$$
P=(a, b) \in K^{2}=\mathbb{A}^{2}(K) \text { with } b^{2}=\prod_{i=1}^{2 g+1}\left(a-\alpha_{i}\right)
$$

the fiber $\mathfrak{h}^{-1}(P)=\mathfrak{R}_{1 / 2, P}$ consists of (familiar) collections of square roots

$$
\mathfrak{r}=\left\{r_{i}=\sqrt{a-\alpha_{i}} \mid 1 \leq i \leq 2 g+1\right\}
$$

with $\prod_{i=1}^{2 g+1} r_{i}=-b$. Each such $\mathfrak{r}$ gives rise to $\mathfrak{a}_{\mathfrak{r}} \in J(K)$ such that

$$
2 \mathfrak{a}_{\mathfrak{r}}=P \in \mathcal{C}(K) \subset J(K)
$$

(see [9, Th. 3.2]). On the other hand, for each $\mathfrak{W}_{l}=\left(\alpha_{l}, 0\right)($ with $1 \leq l \leq 2 g+1)$ the sum $\mathfrak{a}_{\mathfrak{r}}+\mathfrak{W}_{l}$ is also a half of $P$ and therefore corresponds to a certain collection of square roots. Which one? The answer is given by Theorem 1.1. We repeat its statement, using the new notation.

Theorem 4.1. Let $P=(a, b)$ be a K-point on $\mathcal{C}$ and $\mathfrak{r}=\left(r_{1}, \ldots, r_{2 g}, r_{2 g+1}\right)$ be a collection of square roots $r_{i}=\sqrt{a-\alpha_{i}} \in K$ such that $\prod_{i=1}^{2 g+1} r_{i}=-b$. Let $l$ be an integer that satisfies $1 \leq l \leq 2 g+1$ and let

$$
\mathfrak{r}^{[l]}=\left(r_{1}^{[l]}, \ldots, r_{2 g}^{[l]}, r_{2 g+1}^{[l]}\right) \in \mathfrak{h}^{-1}(P) \subset \tilde{\mathcal{C}}(K)
$$

be the collection of square roots $r_{i}^{[l]}=\sqrt{a-\alpha_{i}}$ such that

$$
r_{l}^{[l]}=r_{l}, r_{i}^{[l]}=-r_{i} \forall i \neq l .
$$

Then

$$
\mathfrak{a}_{\mathfrak{r}}+\mathfrak{W}_{l}=\mathfrak{a}_{\mathfrak{r}}[l] .
$$


Example 4.2. Let us take as $P$ the point $\mathfrak{W}_{l}=\left(\alpha_{l}, 0\right)$. Then

$$
r_{l}=\sqrt{\alpha_{l}-\alpha_{l}}=0 \forall \mathfrak{r}=\left(r_{1}, \ldots, r_{2 g}, r_{2 g+1}\right) \in \mathfrak{h}^{-1}\left(\mathfrak{W}_{l}\right)
$$

and therefore

$$
\mathfrak{r}^{[l]}=\left(-r_{1}, \ldots,-r_{2 g},-r_{2 g+1}\right)=-\mathfrak{r} .
$$

It follows from Example 1.3 (if we take $\beta=\alpha_{l}$ ) that

$$
\mathfrak{a}_{\mathfrak{r}}+\mathfrak{W}_{l}=\mathfrak{a}_{\mathfrak{r}}-\mathfrak{W}_{l}=\mathfrak{a}_{\mathfrak{r}}-2 \mathfrak{a}_{\mathfrak{r}}=-\mathfrak{a}_{\mathfrak{r}}=\mathfrak{a}_{\mathfrak{r}}[l] .
$$

This proves Theorem 4.1 in the case of $P=\mathfrak{W}_{l}$. We are going to deduce the general case from this special one.

4.3. Before starting the proof of Theorem 4.1, let us define for each collections of signs

$$
\varepsilon=\left\{\epsilon_{i}= \pm 1 \mid 1 \leq i \leq 2 g+1, \prod_{i=1}^{2 g+1} \epsilon_{i}=1\right\}
$$

the biregular automorphism

$$
T_{\varepsilon}: \tilde{\mathcal{C}} \rightarrow \tilde{\mathcal{C}}, z_{i} \mapsto \epsilon_{i} z_{i} \forall i
$$

Clearly, all $T_{\varepsilon}$ constitute a finite automorphism group of $\tilde{\mathcal{C}}$ that leaves invariant every $K$-fiber of $\mathfrak{h}: \tilde{\mathcal{C}} \rightarrow \mathcal{C} \backslash\{\infty\}$, acting on it transitively. Notice that if $T_{\varepsilon}$ leaves invariant all the points of a certain fiber $\mathfrak{h}^{-1}(P)$ with $P \in \mathcal{C}(K)$ then all the $\epsilon_{i}=1$, i.e., $T_{\varepsilon}$ is the identity map.

Proof of Theorem 4.1. Let us put

$$
\beta:=\alpha_{l} .
$$

Then we have

$$
\mathfrak{W}_{l}=\left(\alpha_{l}, 0\right)=(\beta, 0) .
$$

Let us consider the automorphism (involution)

$$
\mathfrak{s}^{[l]}: \tilde{\mathcal{C}} \rightarrow \tilde{\mathcal{C}}, \mathfrak{r} \mapsto \mathfrak{r}^{[l]}
$$

of $\tilde{\mathcal{C}}$ defined by (6) and (7). We need to define another (actually, it will turn out to be the same) involution (and therefore an automorphism)

$$
\mathfrak{t}^{[l]}: \tilde{\mathcal{C}} \rightarrow \tilde{\mathcal{C}}
$$

that is defined by

$$
\mathfrak{a}_{\mathfrak{t}^{[l]}(\mathfrak{r})}=\mathfrak{a}_{\mathfrak{r}}+\mathfrak{W}_{l}
$$

as a composition of the following regular maps. First, $\mathfrak{r} \in \tilde{C}(K)$ goes to the pair of polynomials $\left(U_{\mathfrak{r}}(x), V_{\mathfrak{r}}(x)\right)$ as in Remark 2.2, which is the Mumford representation of $\mathfrak{a}_{\mathfrak{r}}$ (see Subsect. 2.4). Second, $\left(U_{\mathfrak{r}}(x), V_{\mathfrak{r}}(x)\right)$ goes to the pair of polynomials $\left(U^{[\beta]}(x), V^{[\beta]}(x)\right)$ defined by formulas (3) and (3) in Section 3, which is the Mumford representation of $\mathfrak{a}_{\mathfrak{r}}+\mathfrak{W}_{l}$. Third, applying formulas (1) and (2) in Remark 2.2 to $\left(U^{[\beta]}(x), V^{[\beta]}(x)\right)$ (instead of $\left.(U(x), V(x))\right)$, we get at last $\mathfrak{t}^{[l]}(\mathfrak{r}) \in \tilde{\mathcal{C}}(K)$ such that

$$
\mathfrak{a}_{\mathfrak{t}[l](\mathfrak{r})}=\mathfrak{a}_{\mathfrak{r}}+\mathfrak{W}_{l} .
$$

Clearly, $\mathfrak{t}^{[l]}$ is a regular selfmap of $\tilde{\mathcal{C}}$ that is an involution, which implies that $\mathfrak{t}^{[l]}$ is a biregular automorphism of $\tilde{\mathcal{C}}$. It is also clear that both $\mathfrak{s}^{[l]}$ and $\mathfrak{t}^{[l]}$ leave invariant every fiber of $\mathfrak{h}: \tilde{\mathcal{C}} \rightarrow \mathcal{C} \backslash\{\infty\}$ and coincide on $\mathfrak{h}^{-1}\left(\mathfrak{W}_{l}\right)$, thanks to Example 4.2. This implies that $\mathfrak{u}:=\left(\mathfrak{s}^{[l]}\right)^{-1} \mathfrak{t}^{[l]}$ is a biregular automorphism of $\tilde{\mathcal{C}}$ that leaves 
invariant every fiber of $\mathfrak{h}: \tilde{\mathcal{C}} \rightarrow \mathcal{C} \backslash\{\infty\}$ and acts as the identity map on $\mathfrak{h}^{-1}\left(\mathfrak{W}_{l}\right)$. The invariance of each fiber of $\mathfrak{h}$ implies that $\tilde{\mathcal{C}}(K)$ coincides with the finite union of its closed subsets $\tilde{\mathcal{C}}_{\varepsilon}$ defined by the condition

$$
\tilde{\mathcal{C}}_{\varepsilon}:=\left\{Q \in \tilde{\mathcal{C}}(K) \mid \mathfrak{u}(Q)=T_{\varepsilon}(Q)\right\} .
$$

Since $\tilde{\mathcal{C}}$ is irreducible, the whole $\tilde{\mathcal{C}}(K)$ coincides with one of $\tilde{\mathcal{C}}_{\varepsilon}$. In particular, the fiber

$$
\mathfrak{h}^{-1}\left(\mathfrak{W}_{l}\right) \subset \tilde{\mathcal{C}}_{\varepsilon}
$$

and therefore $T_{\varepsilon}$ acts identically on all points of $\mathfrak{h}^{-1}\left(\mathfrak{W}_{l}\right)$. In light of arguments of Subsect. $4.3, T_{\varepsilon}$ is the identity map and therefore $\mathfrak{u}$ acts identically on the whole $\tilde{\mathcal{C}}(K)$. This means that $\mathfrak{s}^{[l]}=\mathfrak{t}^{[l]}$, i.e.,

$$
\mathfrak{a}_{\mathfrak{r}}+\mathfrak{W}_{l}=\mathfrak{a}_{\mathfrak{r}[l]} .
$$

4.4. Let $\phi: \mathfrak{R} \rightarrow \mathbb{F}_{2}$ be a function that satisfies $\sum_{\alpha \in \mathfrak{R}} \phi(\alpha)=0$, i.e. $\phi \in\left(\mathbb{F}_{2}^{\mathfrak{R}}\right)^{0}$. Then the finite subset

$$
\operatorname{supp}(\phi)=\{\alpha \in \mathfrak{R} \mid \phi(\alpha) \neq 0\} \subset \mathfrak{R}
$$

has even cardinality and the corresponding point of $J[2]$ is

$$
\mathfrak{T}_{\phi}=\sum_{\alpha \in \mathfrak{R}} \phi(\alpha) \mathfrak{W}_{\alpha}=\sum_{\alpha \in \operatorname{supp}(\phi)} \mathfrak{W}_{\alpha}=\sum_{\gamma \notin \operatorname{supp}(\phi)} \mathfrak{W}_{\gamma} .
$$

Theorem 4.5. Let $\mathfrak{r} \in \mathfrak{R}_{1 / 2, P}$. Let us define $\mathfrak{r}^{(\phi)} \in \mathfrak{R}_{1 / 2, P}$ as follows.

$$
\mathfrak{r}^{(\phi)}(\alpha)=-\mathfrak{r}(\alpha) \forall \alpha \in \operatorname{supp}(\phi) ; \mathfrak{r}^{(\phi)}(\gamma)=\mathfrak{r}(\gamma) \forall \gamma \notin \operatorname{supp}(\phi) .
$$

Then

$$
\mathfrak{a}_{\mathfrak{r}}+\mathfrak{T}_{\phi}=\mathfrak{a}_{\left.\mathfrak{r}^{(}()\right)} .
$$

Remark 4.6. If $\phi$ is identically zero then

$$
\mathfrak{T}_{\phi}=0 \in J[2], \mathfrak{r}^{(\phi)}=\mathfrak{r}
$$

and the assertion of Theorem 4.5 is obviously true. If $\alpha_{l} \in \mathfrak{R}$ and $\phi=\psi_{\alpha_{l}}$, i.e. $\operatorname{supp}(\phi)=\mathfrak{R} \backslash\left\{\alpha_{l}\right\}$ then

$$
\mathfrak{T}_{\phi}=\mathfrak{W}_{l} \in J[2], \mathfrak{r}^{(\phi)}=\mathfrak{r}^{[l]}
$$

and the assertion of Theorem 4.5 follows from Theorem 4.1.

Proof of Theorem 4.5. We may assume that $\phi$ is not identically zero. We need to apply Theorem $4.1 d$ times where $d$ is the (even) cardinality of $\operatorname{supp}(\phi)$ in order to get $\mathfrak{r}^{\prime} \in \Re_{1 / 2, P}$ such that

$$
\mathfrak{a}_{\mathfrak{r}}+\sum_{\alpha \in \operatorname{supp}(\phi)} \mathfrak{W}_{\alpha}=\mathfrak{a}_{\mathfrak{r}^{\prime}}
$$

Let us check how many times do we need to change the sign of each $\mathfrak{r}(\beta)$. First, if $\beta \notin \operatorname{supp}(\phi)$ then we need to change to sign of $\mathfrak{r}(\beta)$ at every step, i.e., we do it exactly $d$ times. Since $d$ is even, the sign of $\mathfrak{r}(\beta)$ remains the same, i.e.,

$$
\mathfrak{r}^{\prime}(\beta)=\mathfrak{r}(\beta) \forall \beta \notin \operatorname{supp}(\phi) .
$$

Now if $\beta \in \operatorname{supp}(\phi)$ then we need to change the sign of $\mathfrak{r}(\beta)$ every time when we add $W_{\alpha}$ with $\alpha \neq \beta$ and it occurs exactly $(d-1)$ times. On the other hand, when 
we add $\mathfrak{W}_{\beta}$, we don't change the sign of $\mathfrak{r}(\beta)$. So, we change the sign of $\mathfrak{r}(\beta)$ exactly $(d-1)$ times, which implies that

$$
\mathfrak{r}^{\prime}(\beta)=-\mathfrak{r}(\beta) \forall \beta \in \operatorname{supp}(\phi) .
$$

Combining the last two displayed formula, we obtained that

$$
\mathfrak{r}^{\prime}=\mathfrak{r}^{(\phi)} .
$$

\section{Useful Lemma}

As usual, we define the Kronecker delta $\delta_{i k}$ as 1 if $i=k$ and 0 if $i \neq k$.

The following result is probably well known but I did not find a suitable reference. (However, see [3, Lemma 5.10] and [1, pp. 425-427].)

Lemma 5.1. Let $n$ be a positive integer, $E$ a field provided with $n$ distinct discrete valuation maps

$$
\nu_{i}: E^{*} \rightarrow \mathbb{Z},(i=1, \ldots, n) .
$$

For each $i$ let $O_{\nu_{i}} \subset E$ the discrete valuation ring attached to $\nu_{i}$ and $\pi_{i} \in O_{\nu_{i}}$ its uniformizer, i.e., a generator of the maximal ideal in $O_{\nu_{i}}$. Suppose that for each $i$ we are given a prime number $p_{i}$ such that the characteristic of the residue field $O_{\nu_{i}} / \pi_{i}$ is different from $p_{k}$ for all $k \neq i$. Let us assume also that

$$
\nu_{i}\left(\pi_{k}\right)=\delta_{i k} \forall i, k=1, \ldots n
$$

i.e, each $\pi_{i}$ is a $\nu_{k}$-adic unit if $i \neq k$.

Then the the quotient $B=E\left[T_{1}, \ldots, T_{n}\right] /\left(T_{1}^{p_{1}}-\pi_{1}, \ldots, T_{n}^{p_{n}}-\pi_{n}\right)$ of the polynomial E-algebra $E\left[T_{1}, \ldots T_{n}\right]$ by the ideal generated by all $T_{i}^{p_{i}}-\pi_{i}$ is a field that is an algebraic extension of $E$ of degree $\prod_{i=1}^{n} p_{i}$. In addition, the set of monomials

$$
S=\left\{\prod_{i=1}^{n} T_{i}^{e_{i}} \mid 0 \leq e_{i} \leq p_{i}-1\right\} \subset E\left[T_{1}, \ldots T_{n}\right]
$$

maps injectively into $B$ and its image is a basis of the E-vector space $B$.

Remark 5.2. By definition of a uniformizer, $\nu_{i}\left(\pi_{i}\right)=1$ for all $i$.

Proof of Lemma 5.1. First, the cardinality of $S$ is $\prod_{i=1}^{n} p_{i}$ and the image of $S$ generates $B$ as the $E$-vector space. This implies that if the $E$-dimension of $B$ is $\prod_{i=1}^{n} p_{i}$ then the image of $S$ is a basis of the $E$-vector space $B$. Second, notice that for each $i$ the polynomial $T^{p_{i}}-\pi_{i}$ is irreducible over $E$, thanks to the Eisenstein criterion applied to $\nu_{i}$ and therefore $E\left[T_{i}\right] /\left(T^{p_{i}}-\pi_{i}\right)$ is a field that is an algebraic degree $p_{i}$ extension of $E$. In particular, the $E$-dimension of $E\left[T_{i}\right] /\left(T^{p_{i}}-\pi_{i}\right)$ is $p_{i}$. This proves Lemma for $n=1$.

Induction by $n$. Suppose that $n>1$ and consider the finite degree $p_{i}$ field extension $E_{n}=E\left[T_{n}\right] /\left(T^{p_{n}}-\pi_{n}\right)$ of $E$.

Clearly, the $E$-algebra $B$ is isomorphic to the quotient $E_{n}\left[T_{1}, \ldots T_{n-1}\right] /\left(T_{1}^{p_{1}}-\right.$ $\left.\pi_{1}, \ldots, T_{n-1}^{p_{n-1}}-\pi_{n-1}\right)$ of the polynomial ring $E_{n}\left[T_{1}, \ldots T_{n-1}\right]$ by the ideal generated by all polynomials $T_{i}^{p_{i}}-\pi_{i}$ with $i<n$. Our goal is to apply the induction assumption to $E_{n}$ instead of $E$. In order to do that, let us consider for each $i<n$ the integral closure $\tilde{O}_{i}$ of $O_{\nu_{i}}$ in $E_{n}$. It is well known that $\tilde{O}_{i}$ is a Dedekind ring. Our conditions imply that $E_{n} / E$ is unramified at all $\nu_{i}$ for all $i<n$. This means 
that if $\mathcal{P}_{i}$ is a maximal ideal of $\tilde{O}_{i}$ that contains $\pi_{i} \tilde{O}_{i}$ (such an ideal always exists) and

$$
\operatorname{ord}_{\mathcal{P}_{i}}: E_{n}^{*} \rightarrow \mathbb{Z}
$$

is the discrete valuation map attached to $\mathcal{P}_{i}$ then the restriction of $\operatorname{ord}_{\mathcal{P}_{i}}$ to $E^{*}$ coincides with $\nu_{i}$. This implies that for all positive integers $i, k \leq n-1$

$$
\operatorname{ord}_{\mathcal{P}_{i}}\left(\pi_{k}\right)=\nu_{i}\left(\pi_{k}\right)=\delta_{i k} .
$$

In particular,

$$
\operatorname{ord}_{\mathcal{P}_{i}}\left(\pi_{i}\right)=\nu_{i}\left(\pi_{i}\right)=1,
$$

i.e, $\pi_{i}$ is a uniformizer in the corresponding discrete valuation (sub)ring $O_{\operatorname{ord}_{\mathcal{P}_{i}}}$ of $E_{n}$ attached to $\operatorname{ord}_{\mathcal{P}_{i}}$. Now the induction assumption applied to $E_{n}$ and its $(n-1)$ discrete valuation maps $\operatorname{ord}_{\mathcal{P}_{i}}(1 \leq i \leq n-1)$ implies that $B / E_{n}$ is a field extension of degree $\prod_{i=1}^{n-1} p_{i}$. This implies that the degree

$$
[B: E]=\left[B: E_{n}\right]\left[E_{n}: E\right]=\left(\prod_{i=1}^{n-1} p_{i}\right) p_{n}=\prod_{i=1}^{n} p_{i} .
$$

This means that the $E$-dimension of $B$ is $\prod_{i=1}^{n} p_{i}$ and therefore the image of $S$ is a basis of the $E$-vector space $B$.

Corollary 5.3. We keep the notation and assumptions of Lemma 5.1. Let $R$ be a subring of $E$ that contains 1 and all $\pi_{i}(1 \leq i \leq n)$. Then the quotient $B_{R}=$ $R\left[T_{1}, \ldots, T_{n}\right] /\left(T_{1}^{p_{1}}-\pi_{1}, \ldots, T_{n}^{p_{n}}-\pi_{n}\right)$ of the polynomial $R$-algebra $R\left[T_{1}, \ldots, T_{n}\right]$ by the ideal generated by all $T_{i}^{p_{i}}-\pi_{i}$ has no zero divisors.

Proof. There are the natural homomorphisms of $R$-algebras

$$
R\left[T_{1}, \ldots T_{n}\right] \rightarrow B_{R} \rightarrow B
$$

such that the first homomorphism is surjective and the injective image of

$$
S \subset R\left[T_{1}, \ldots T_{n}\right] \subset E\left[T_{1}, \ldots T_{n}\right]
$$

in $B$ is a basis of the $E$-vector space $B$. On the other hand, the image of $S$ generates $B_{R}$ as $R$-module. It suffices to prove that $B_{R} \rightarrow B$ is injective, since $B$ is a field by Lemma 5.1.

Suppose that $u \in B_{R}$ goes to 0 in $B$. Clearly, $u$ is a linear combination of (the images of ) elements of $S$ with coefficients in $R$. Since the image of $u$ in $B$ is 0 , all these coefficients are zeros, i.e., $u=0$ in $B_{R}$.

Example 5.4. We use the notation of Section 4. Let us put $n=2 g+1, R=$ $K[x], E=K(x), \pi_{i}=x-\alpha_{i}, p_{i}=2$ and let

$$
\nu_{i}: E^{*}=K(x)^{*} \rightarrow \mathbb{Z}
$$

be the discrete valuation map of the field of rational functions $K(x)$ attached to $\alpha_{i}$. Then $K[\tilde{\mathcal{C}}]=B_{R} / \mathcal{N}\left(B_{R}\right)$ where $\mathcal{N}\left(B_{R}\right)$ is the nilradical of $B_{R}$. It follows from Corollary 5.3 that $\mathcal{N}\left(B_{R}\right)=\{0\}$ and $K[\tilde{\mathcal{C}}]$ has no zero divisors, i.e., $\tilde{\mathcal{C}}$ is irreducible. 


\section{REFERENCES}

[1] T. Bandman, S. Garion, F. Grunewald, On the surjectivity of Engel words on PSL $(2, q)$. Groups Geom. Dyn. 6 (2012), 409-439.

[2] B.M. Bekker, Yu.G. Zarhin, The divisibility by 2 of rational points on elliptic curves. Algebra i Analiz 29:4 (2017), 196-239; St. Petersburg Math. J. 29 (2018), 683-713.

[3] N. Bruin and E.V. Flynn, Towers of 2-covers of hyperelliptic curves. Trans. Amer. Math. Soc. 357 (2005), no. 11, 4329-4347.

[4] D. Mumford, Tata Lectures on Theta. II. Progress in Math. 43, Birkhäuser, Boston Basel Stutgart, 1984.

[5] M. Stoll, Arithmetic of Hyperelliptic Curves. Available at Summer Semester 2014, University of Bayreuth. http://www.mathe2.uni-bayreuth.de/stoll/teaching/ArithHypKurvenSS2014/Skript-ArithHypCurves-pub-screen.pdf .

[6] E. Schaefer, 2-descent on the Jacobians of hyperelliptic curves. J. Number Theory 51 (1995), no. 2, 219-232.

[7] J.-P. Serre, Algebraic groups and class fields. Graduate Texts in Math. 117, Springer-Verlag, New York, 1988.

[8] L.C. Washington, Elliptic Curves: Number Theory and Cryptography. Second edition. Chapman \& Hall/CRC Press, Boca Raton London New York, 2008.

[9] Yu. G. Zarhin, Division by 2 on odd degree hyperelliptic curves and their jacobians. Izvestiya RAN 83:3 (2019), 93-112; Izvestiya Mathematics 83:3 (2019), 501-520.

Pennsylvania State University, Department of Mathematics, University Park, PA 16802, USA

E-mail address: zarhin@math.psu.edu 